\title{
Performance Analysis of Burst Segmentation Schemes Supporting Multiple Traffic Classes $^{1}$
}

\author{
K. S. Chan \\ Department of Electrical and Computer Engineering \\ Curtin University of Technology \\ Perth, WA, 6845 Australia \\ chank@ece.curtinedu,au
}

\author{
Kwan. L. Yeung \\ Department of Electrical and Electronic Engineering \\ The University of Hong Kong \\ Hong Kong \\ kyeung@eee.hku.hk
}

\begin{abstract}
Optical burst switching (OBS) with the support of multiple traffic classes is an important topic. Assuming traffic classes are maintained via choosing different offset times, a new OBS scheme called optical burst switching with burst splitring (OBSS) is proposed in this paper. OBSS provides high system throughput by taking advantages of the idle gaps on output wavelength channels when the Just-EnoughTime (JET) reservation scheme is used. Then an analytical model that supports $N$ traffic classes is constructed and verified by simulations. The proposed model can be applied to the conventional OBS schemes as well as the OBSS scheme we proposed. Numerical results show that in our OBSS scheme, traffic classes can be effectively maintained via different offset times, and the packet loss probability is also much smaller than the conventional OBS. It is interesting to find that the choice of offset time for a specific traffic class affects the packet loss probabilities of classes higher than it, while the impact to classes below it is much smaller.
\end{abstract}

\section{Introduction}

Optical burst switch (OBS) has been proposed as an efficient switching technique to exploit the terabit bandwidth of WDM networks [1-6]. The jdea underlying OBS technology is to decouple the data path from the control path. In particular, IP packets are aggregated into much larger data bursts before transmission through the OBS network. This reduces the overhead of switching many small packets individuatly. Each data burst is preceded by a control packet which is sent over a separate control wavelength channel. The time difference between sending the control packet and the associated data burst is known as the offset fime. The control packet requests a one-way wavelength resource allocation along the identified path. Several reservation protocols have been proposed in the literature, including JIT (Just-In-Time) [3], Horizon [4] and JET (Just-Enough-Time) [6]. In this paper, we adopt JET, which reserves only the time interval where the data burst will occupy. The starting and ending times of a data burst is carried in the control packet, so it can be known in advance.

OBS faces two challenging issues, how to reduce the packet loss rate due to failed one-way reservation, and how to support different classes of services. To reduce the packet loss rate, an effective mechanism is burst segmentation, which is proposed in [7] and analyzed in [8-10] for supporting a single traffic class, i.e., the offset times for all bursts are the same. In the optical composite burst switch (OCBS) [7] scheme, when a burst arrives and finds that all output wavelength channels are busy, the first part of the burst will be dropped. If one wavelength channel becomes idle before the burst finishes, the remaining portion of the burst will be switched to use this idle output wavelength channel. The scheme works well if only one service class is supported.

If different offset times are employed for bursts belonging to different traffic classes, we may observe some idle gaps on the output wavelength channels. If JET is used, the durations of those idle gaps are known in advance. If we allow a data burst to be split into segments and each segment to be switched to a different output wavelength channel, the packet loss ratio can be reduced. This is the basic idea of our optical burst switching with burst splitting (OBSS) scheme. Please refer to Section 2 for more details.

Another important issue of OBS is to support different classes of traffic. It is proposed in [11] that the service classes can be maintained by choosing different offset times. The burst loss probability of a service class with larger offset time is lower than that of the service class with smaller offset time. The analytical model for an OBS supporting two service classes is proposed in [12] and its accuracy is verified by simulations. Our second major contribution in this paper is to extend this analytical model to a more general case where any number of service classes are supported. We show that our new model can be applied to conventional OBS as well as the new OBSS scheme. The accuracy is again verified by simulations.

Numerical resulis show that the packet loss probability of OBSS is much lower than that of the conventional OBS, and the service classes are effectively maintained by adopting different offset times. We find that for a particular service class, increasing its offset time can reduce the packet loss probability for this service class. Although this gain is at the expenses of higher packet loss probability for traffic belonging to higher service classes, the impact to traffic of lower classes is much smaller.

The remaining of this paper is organized as follows. In Section 2, we explain the operation of our new OBSS scheme. The analytical model is constructed in Section 3. Both numerical and simulation results are presented in

${ }^{1}$ This work was supported by the Research Grants Council of Hong Kong (RGC) under Grant No. 7032/01E. 
Section 4 for performance evaluations. In Section 5, we conclude the paper.

\section{Optical Burst Switch with Burst Splitting (OBSS)}

Fig. 1shows the optical burst switch architecture, which is the same as the one proposed in [7]. To keep the paper concise, we skip the introduction of the switch architecture and only focus on its operations. Readers not familiar with this architecture please refer to [7].

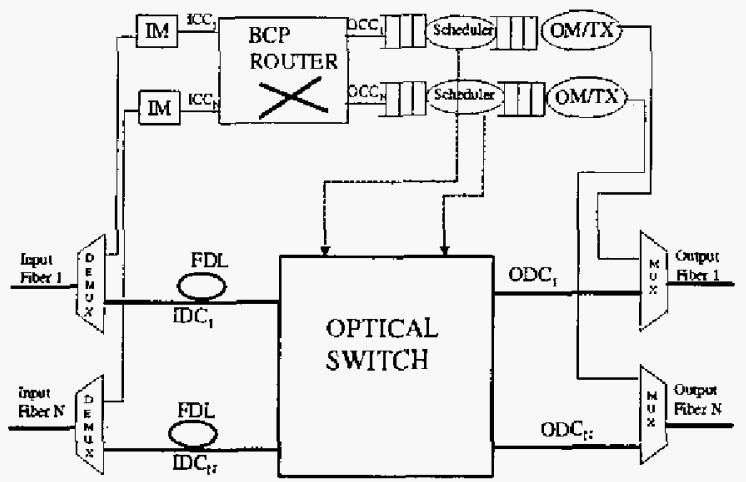

Fig. 1Optical burst switch with burst splitting (OBSS) architecture

Each data burst is preceded by a burst control packet which is sent over the control wavelength channel. The offset time between the control packet and the associated data burst determines the service class this burst belongs to. If the offset time is large, the chance of successful resontce reservation is then high. Passing through the demultiplexer in Fig. 1, the control packet is delivered to the electronic control plane. The control packet carries the routing information as well as the starting and ending times of the associated data burst. In the control plane, the scheduler checks if the whole data burst can be switched (thus carried by) onto an idle data wavelength channel. Based on the initial scheduling result, there are two operation modes:

(1) Mode 1: switching without splitting. If the scheduler can find an output wavelength channel that is idle for the whole time interval of the data burst, resource reservation is successful and the resulting schedule is stored for proper switch fabric configuration later on. In this case, the data burst can be switched without splitting.

(2) Mode 2: switching with splitting. If no output wavelength channel can accommodate the whole data burst, the scheduler examines the idle gaps on all wavelength channels during the requested burst interval. If sufficient idle gaps are found to cover the burst, the scheduler will split the burst into segments, one for each gap. The corresponding schedule is then stored for properly configuring the optical switch. The segmentation procedure for a data burst is explained in [7]. Noted that each successfully split segment must be preceded by a dedicated control packet.
The detailed operations of our OBSS scheme can be illustrated using the example shown in Fig. 2. Consider an arbitrary output port of an optical burst switch which has two data and one control wavelength channels. At time $t_{0}$, assume output wavelength channel $2\left(\mathrm{ODC}_{2}\right)$ is busy until time $t_{3}$ and $\mathrm{ODC}_{1}$ is idle. $A$ control packer arrives on channel ICC of an input port at time $t_{0}$, whose data burst starts at time $t_{3}$ and ends at time $t_{5}$ (as shown in $\mathrm{WC}_{1}$ ). As time interval $\left[t_{3}, t_{5}\right]$ on channel $O \mathrm{OC}_{1}$ is idle, it is reserved by this control packet. In this case, the arriving burst will be switched using Mode 1 switching without splitting. Please notice that the offset time of this burst is $t_{3}-\tau_{0}$.

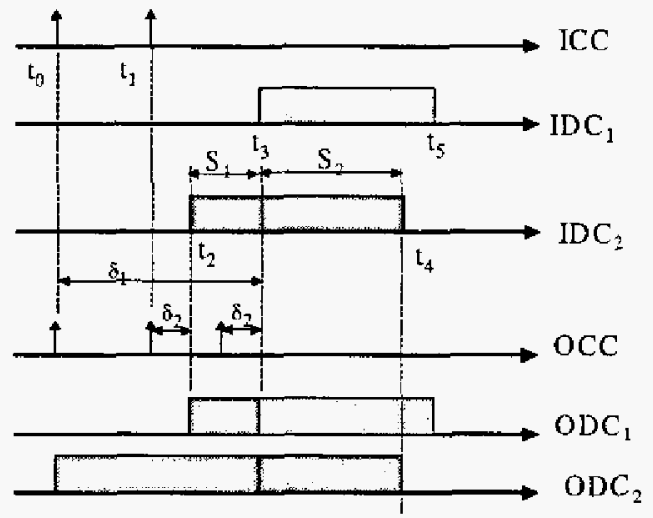

ICC: input control channel IDC: input data channel OCC: output control channel ODC: output data channel

Fig. 2Burst splitting technique with two output data wavelength channels.

Assume the second control packet arrives at the same input control channel at time $t_{1}$ for a data burst starting at time $t_{2}$ $\left(<t_{3}\right)$ and ending at $t_{4}\left(<t_{5}\right)$. As no output data channel is idle for the whole time interval $\left[t_{2}, t_{4}\right]$, this burst cannot be switched without splitting. We try Mode 2. It can be seen that for time interval $\left[t_{2}, t_{3}\right]$, output channel $O D C_{1}$ is idle, and for interval $\left[t_{3}, t_{4}\right], O D C_{2}$ is idle. Therefore, we can deliver the whole burst by splitting it into two segments, segment 1 from $t_{2}$ to $t_{3}$ over $O D C_{1}$ and segment 2 from $t_{3}$ to $\mathrm{t}_{4}$ over $\mathrm{ODC}_{2}$.

Although burst segmentation/splitting can reduce the burst loss probability, additional overhead for inserting and modifying control packets is introduced. We believe this overhead can be absorbed/solved quite easily as the control packet is processed in electrical domain. Comparing our scheme with the existing burst segmentation scheme OCBS [7], it can be seen that the first segment of the second data burst in our example above will be dropped in OCBS. Therefore, intuitively the packet loss probability of our OBSS scheme is lower than OCBS.

\section{Analytical Model}

In this section, we construct an analytical model to evaluate our proposed OBSS scheme. Without loss of generality, we consider an OBS node that supports $N$ service classes, labeled from 1 to $N$, with class $N$ the highest. For each output port, we have $k$ wavelength channels. Wavelength conversions are allowed if necessary. We focus on the packet loss probability of a tagged output port. We assume 
that the switch size is large enough, such that we can model the class $i$ burst arrival process at the tagged output port by a Poisson process with rate $\lambda_{i}$. We also assume that for class $i$ traffic, the burst length is exponentially distributed with mean $\mu_{i}$, and the offset time equals to $\delta_{i}$. For simplicity, let $\delta_{1}=0$. As in [9], we further assume that packets are small relative to the burst, and bursts are segmented at the boundary of packets. Therefore, packet loss due to the segmentation in the middle of a packet is negligible.

Let $A_{i}=\lambda_{i} \mu_{i}$ and $A_{\text {oll }}=\Sigma A_{i} . A_{i}$ represents the offered load to the tagged output port from class $i$, and $A_{\text {all }}$ is the total offered load to the tagged output port. We first derive the equation of the packet loss probability given that the offered load is $A$. If OBSS is used and the number of available output wavelength channels is $k$, the switching can be modeled by a $\mathrm{M} / \mathrm{G} / \propto$ queue. Following the derivation in [9], we have the packet loss probability

$$
P_{\text {lass }}(A)=E[L] / A
$$

where

$$
\begin{aligned}
& E[L]=\sum_{i=1}^{\infty} i P(k+i) \\
& P(k+i)=A^{k+i} \frac{e^{-A}}{(k+i) !}
\end{aligned}
$$

Let $P_{\text {loss.i }}$ be the packet loss probability of service class $i$ traffic, $P_{\text {loss all }}$ be the overall packet loss probability for all $N$ traffic classes, and $P_{\text {loss, } t k}$ be the total packet loss probability for all classes higher than or equal to $k$. To understand $P_{\text {loss, }, k}$, consider a system supporting 10 classes, from 1 to 10. $P_{\text {loss. } .7}$ is the overall packet loss probability of the group consisting of service classes $7,8,9$ and 10 . It can also be easily shown that $P_{\text {loss all }}=P_{\text {lass }}\left(A_{\text {all }}\right)$.

Next we derive the packet loss probability for service class $N$. Let $Y_{j}\left(\delta_{N}\right)$ represent the portion of class $j(j<N)$ traffic which will affect the packet loss probability of service class $N$. In other words, those are the class $j$ packets with their control packets arrived prior to the arrival of the class $N$ control packet, and they are served after the class $N$ burst starts, i.e., $\delta_{N}$ after the class $N$ control packet has arrived. This additional traffic stems from the fact that high class traffic is not totally isolated from low class traffic in our system. Then we have

$$
P_{l o s s, N}=P_{\text {loss }}\left(A_{N}+\sum_{j=1}^{N-1} Y_{j}\left(\delta_{N}\right)\right)
$$

where

$$
Y_{j}\left(\delta_{N}\right)=\lambda_{j}\left(1-P_{\text {lass } . j}\right) \int_{\delta_{N}-\delta_{j}}^{\infty}\left(x-\delta_{N}+\delta_{j}\right) f_{j}(x) d x
$$

$f_{f}(x)$ is the probability density function of the burst length distributjon for service class $j$. It should be pointed out here that we treat the residual traffic $Y_{j}\left(\delta_{N}\right)$ as following a Poisson process, which may introduce some inaccuracy to our analytical model. As seen from the comparison between analytical and simulation results fater, the inaccuracy introduced by this assumption is acceptable.
The integral part in Eqn. (4) is the portion of class $j$ traffic which will be served after $\delta_{N}$ since the control packet of this class $j$ burst arrives. This equation can be explained based on Fig. 3. Assuming that the control packet of class $j$ burst arrives at time $t_{j}$. The associated data burst will arrive at time $t_{j}+\delta$. Now consider the impact of this burst to the packet loss probability of class $N$ traffic. As only class $N$ bursts with the arrival time of control packet after $t_{j}$ will be affected, and the earliest burst arrival time from class $N$ is $t_{j}+\delta_{N}$, then only class $j$ bursts whose service times are after $t_{j}+\delta_{k}$ should be considered. As this class $j$ burst starts at time $t_{j}+\delta_{j}$, and the burst length distribution density function is $f_{j}(x)$, the portion of traffic to be served after time $t_{j}+\delta_{N}$ is

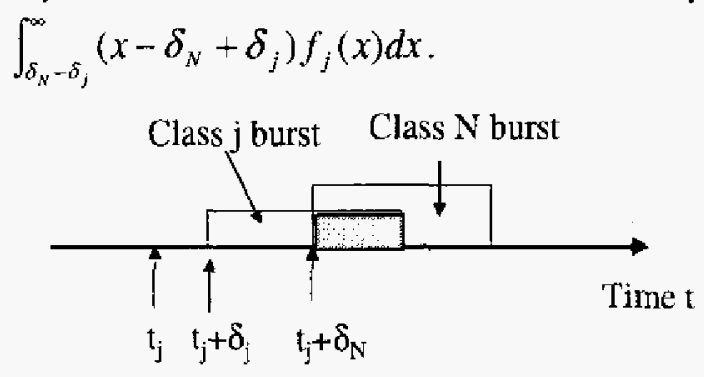

Fig. 3 The impact of class $j$ traffic to the packet loss probability of class $N$ traffic

Similarly, we can derive the general expression for $Y_{j}\left(\delta_{k}\right)$, the portion of traffic from class $j$ which will affect the packet loss ratio of class $k>j$.

$$
Y_{j}\left(\delta_{k}\right)=\lambda_{j}\left(1-P_{l o s s, j}\right) \int_{\delta_{k}-\delta_{j}}^{\infty}\left(x-\delta_{k}+\delta_{j}\right) f_{j}(x) d x
$$

If the burs: length is exponentially distributed, we have

then

$$
f_{j}(x)= \begin{cases}\frac{1}{\mu_{j}} e^{-\frac{x}{\mu_{j}}}, & \text { if } x \geq 0 \\ 0, & \text { if } x<0\end{cases}
$$

$$
Y_{j}\left(\delta_{k}\right)=\lambda_{j} \mu_{j}\left(1-P_{\text {lass }, j}\right) k^{\frac{\delta_{j}-\delta_{k}}{\mu_{j}}}
$$

Now we try to get the packet loss probability for class $k$ traffic. We first consider $P_{\text {loss, }, k \text {, }}$, the overall packet loss probability for all classes greater than or equal to $k$. We have

$$
P_{\text {loss }, t k}=P_{\text {loss }}\left(\sum_{i=k}^{N} A_{i}+\sum_{j=1}^{k-1} Y_{j}\left(\delta_{k}\right)\right)
$$

According to the conservation law, we have

$$
\left(\sum_{i=k}^{N} A_{i}+\sum_{j=1}^{k-1} Y_{j}\left(\delta_{k}\right)\right) P_{\text {loss }, k}=\sum_{i=k}^{N} A_{i} P_{\text {loss } . i}
$$

From (3), we get the packet loss probability for class $N$. From (6), we have $P_{\text {lors, }\{(N-1) \text {, the overall packet loss }}$ probability for classes $N-1$ and $N$. Then from (7), we can have the packet loss probability for class $N-1$. Following similar steps, we can have the packet loss probability for class $N-2$, and so on. As a result, we can obtain packet loss probability for any traffic class. 
The above set of equations can be solved iteratively, with the following initial values

$$
\begin{aligned}
& P_{\text {loss }, N}^{(0)}=P_{\text {loss }}\left(A_{N}\right) \\
& P_{\text {loss }, \mathrm{kk}}^{(0)}=P_{\text {loss }}\left(\sum_{i=k}^{N} A_{i}\right)
\end{aligned}
$$

It should be noticed that our analytical model can be easily modified to study the performance of conventional OBS with different traffic classes. As burst segmentation is not supported in conventional optical burst switching, we should use $\mathrm{M} / \mathrm{M} / \mathrm{k} / \mathrm{k}$ queue to model the switch when we derive the overall packet loss probability. As shown in [9], we can simply use the following $P_{\text {loss }}^{\text {COBS }}(k, A)$ expression to replace $\mathrm{P}_{\text {loss }}(\mathrm{A})$ in (1):

$$
\left\{\begin{array}{c}
P(0, A)=1 \\
P(n, A)=\frac{A P(n-1)}{n+A P(n-1)}, \quad \mathrm{n}=1,2,3, \cdots, \mathrm{k}
\end{array}\right.
$$

Then we can solve all equations iteratively and obtain the packet loss probabilities for all service classes.

\section{Performance Evaluations}

In this section, we consider a three-class traffic scenario to demonstrate the performance of our proposed OBSS scheme. The three classes are labeled as high, medium and low. We assume that the burst size distributions for all three classes are i.i.d. and exponentially distributed with mean $\mu$. In all the figures shown below, we assume that $20 \%$ of the incoming traffic belongs to high service class, $30 \%$ belongs to medium service class, and the remaining $50 \%$ belongs to low service class.

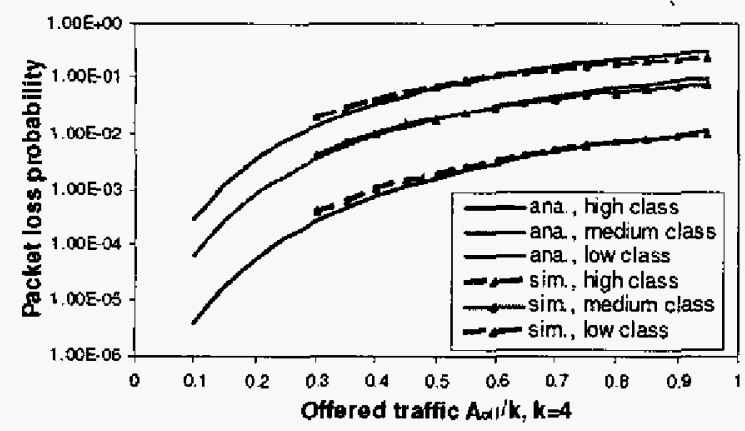

Fig. 4 Packet loss probability versus traffic load for OBSS, with of fset times $2 \mu, \mu$, and 0 , respectively

Given that each fiber supports $k=4$ wavelength channels, Fig. 4 shows the packet loss probability for different traffic classes versus the total traffic load. The offset times for high, medium, and low service classes are $2 \mu, \mu$, and 0 , respectively. Both simulation and analytical results are presented in Fig. 4. It can be seen that the simulation results match quite well with the analytical results. The minor performance difference stems from the Poisson assumption we have made on the residual traffic. It can also be seen that packet loss probabilities for all three classes increase as the loat increases, while the packet loss probability of high service class is much smaller than that of medium service class, and the packet loss probability of low service class is the highest. This shows that service classes can be effectively maintained in our OBSS scheme.

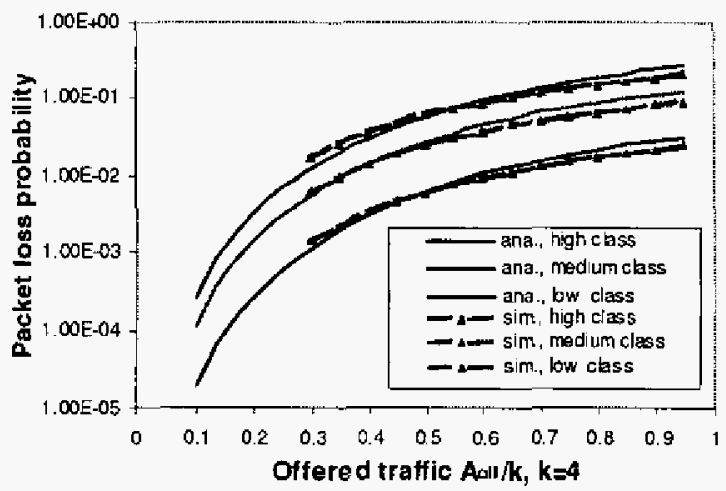

Fig. 5 Packet loss probability versus traffic load for OBSS, with offset times $\mu, \mu / 2$, and 0 , respectively

Fig. 5 shows the packet loss performance with another set of offset times, $\mu, \mu / 2$, and 0 , for high, medium and low classes, respectively. Again simulation results match quite well with our analytical results. Compared with Fig. 4, we can see that the packet loss probabilities for both high class and medium class traffic increase, while the packet loss probability for low class traffic reduces slightly. This verifies that if we increase the offset time for a particular service class, lower packet loss probability for that service class can be achieved.

In Fig. 6, we use our analytical model to analyze the performance of the conventional optical burst switching (i.e. no burst splitting, or OBSS operates only in Mode 1). The offset times for the three classes are $2 \mu, \mu$, and 0 , respectively. Simulation results again match well with our analytical results. Comparing with the performance of our OBSS in Fig. 4, we can see that packet loss probabilities for all three classes are increased. This is because that the overall packet loss probability for OBSS is lower than that withour splitting.

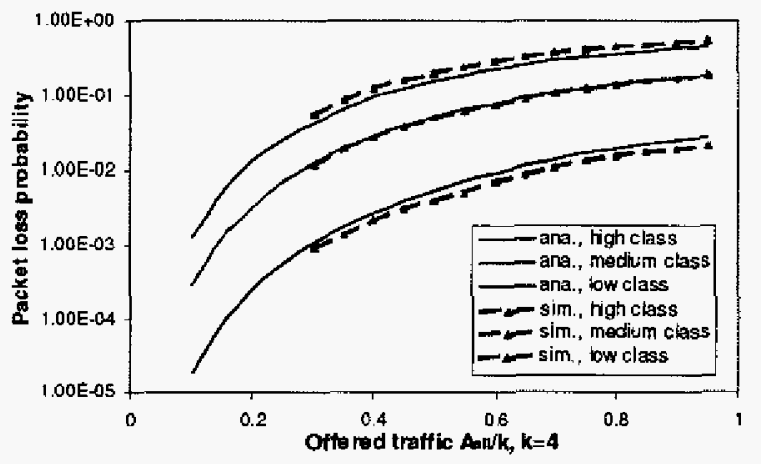

Fig, 6 Packet loss probability versus traffic load for conventional optical burst switch

Fig. 7 shows the packet loss probability of OBSS by varying the number of wavelengths supported on each fiber from $k=8$ to 64 . The offset times for the three classes are $\mu, \mu / 2$, 
and 0 , respectively. As expected, when $k$ increases the packet loss probability drops.

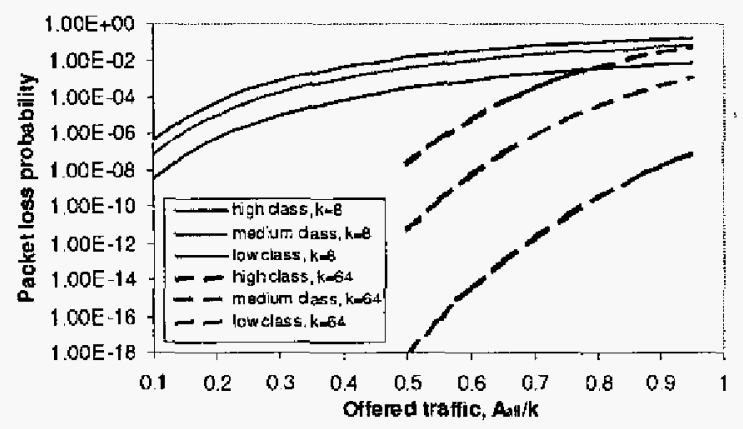

Fig. 7 Packet loss probability versus traffic load for OBSS, when $\mathrm{k}=8$ or 64 , with offset times $\mu, \mu / 2$, and 0 , respectively

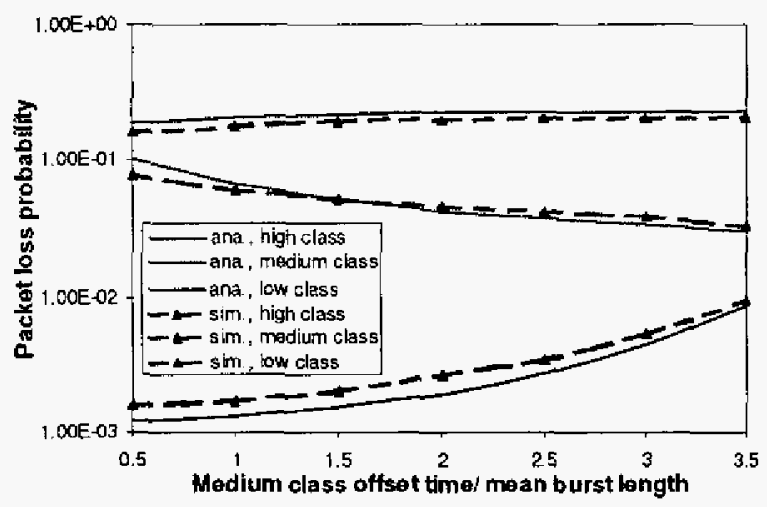

Fig. 8 Packet loss probability versus medium class traffic offset time, $k=4$

Fig. 8 shows the impact of varying the offset time for medium class traffic on the packet loss probabilities of the other two classes. The offset time for medium class changes from $0.5 \mu$ to $3.5 \mu$, while the offset time for high class is kept at $4 \mu$. The total offered traffic load is $A_{a l} / k=0.8$ and $k=4$. It can be seen that the packet loss probability for low service class remains more or less the same. The packet loss probability for medium service class decreases as the offset time increases. This matches the intuition that the larger offset time a service class has, the lower packet loss probability it will get. Nevertheless, the packet loss probability of high service class increases when the offset time for medium service class increases. It is because that if the overall incoming traffic load is the same, the overall packet loss probability remains unchanged. Then the reduction of packet loss probability in medium service class is at the expense of higher packet loss probability in high service class. This observation is also true for conventional optical burst switch supporting different traffic classes.

\section{Conctusions}

In this paper, we observed that due to the offset time difference at an optical burst switch, idle gaps on the output wavelength channels can be found. When the Just-EnoughTime (JET) scheme is used for data burst reservation, these idle gaps can be known in advance. To minimize the packet loss probability, we proposed a new burst segmentation scheme, called optical burst switching with burst splitting (OBSS) which takes advantages of these known idle gaps. Then an analytical model for supporting $N$ service classes is constructed, where each class is identified by a different offset time. The proposed analytical model can be applied to the conventional optical burst switching (OBS) as well as the OBSS scheme we proposed. Simulation results are also presented and they match very well with our analytical results which verifies our model. Numerical results show that in our OBSS scheme, traffic classes can be effectively maintained via different offset times, and the packet loss probability is also much smaller than the conventional OBS. It is also interesting to find that the choice of offset time for a specific traffic class will affect the packet loss probabilities of higher service classes, while the impact to lower service classes is much smaller.

\section{References}

[1] C. Qiao and M. Yoo, "Optical burst switching (OBS) - a new paradigm for an optical internet", Journal of High Speed Networks, vol. 8, no. 1, pp. 69-84, 1999.

[2] S. Verma, H. Chaskar and R. Ravikanth, "Optical burst switching: a viable solution for terabit $\mathrm{T}$ backbone", IEEE Network, pp. 48-53, 2000.

[3] J. Y. Wei, J. L. Pastor, R. S. Ramamurthy and Y. Tsai, "Just-in-time optical burst switching for multi-wavelength networks", Proceedings, $5^{\text {th }}$ Int. Conf. Broadband Comm., pp. 339-352, 1999.

[4] Y. Chen and J. Tumer, "WDM burst switching for petabit capacity routers", Proceedings, MILCOM, vol. 2, pp. 968-973, 1999.

[5] Y. Xiong, M. Vandenhoute and H. Cankaya, "Control architecture in optical burst-switched WDM networks", IEEE JSAC, vol. 18, no. 10, pp. 1838-1851, 2000.

[6] C. Qiao, "Labeled optical burst switching for IP-overWDM integration", IEEE Commun. Mag., vol. 38, no. 9, pp. 104-114, 2000.

[7] A. Detti, V. Eramo and M. Listanti, "Performance evaluation of a new technique for IP support ina WDM optical network: optical composite burst switching (OCBS)", Journal of lightwave technology, vol. 20, no. 2, pp. 154-165, 2002.

[8] V. Vokkarane, J. Jute and S. Sitaraman, "Burst segmentation: an approach for reducing packet loss in optical burst switched networks", Proceedings, ICC'O2, pp. 2673-2677, 2002.

[9] M. Neuts, Z. Rosberg, H. Vu, J. White and M. Zukerman, "Performance analysis of optical composite burst switching", IEEE Commu. Letters, vol. 6, no. 8, pp. 346348,2002 .

[10] Z. Rosberg, H. Vu and M. Zukerman, "Burst segmentation benefit in optical switching", IEEE Commu. Letters, vol. 7, no. 3, pp. 127-129, 2003.

[11] M. Yoo and C. Qiao, "Supporting multiple classes of services in IP over WDM networks", Proceedings, Globecom'99, pp. 1023-1027, 1999.

[12] K. Dolzer, C. Gauger, J. Spath and S. Bodamer, "Evaluation of reservation mechanisms for optical burst switching", AEU Int. J. Electron. Commun., pp. 1-8, 2001. 